\title{
An integrated location inventory routing model in supply chain network designing under uncertainty
}

\author{
Hojat Angazi*
}

Department of industrial engineering, Firoozkooh branch, Islamic azad university, Firoozkooh, iran

\begin{tabular}{l}
\hline C H R O N I C L E \\
\hline Article history: \\
Received February 25, 2016 \\
Received in revised format: \\
March 28, 2016 \\
Accepted March 31, 2016 \\
Available online \\
April 5 2016 \\
\hline Keywords: \\
Inventory location problem \\
Routing \\
Disruption risk \\
Partial Backorder \\
Outer approximation
\end{tabular}

\section{Introduction}

The disruption risks such as earthquakes, hurricanes, or accidents are events with low probability of occurrence but severe effects. Supply chain disruptions have major impacts on the performance of organizations (Huang et al., 2006). Therefore, there is a need for appropriate and effective strategies in order to reduce existing risks and to have a supply chain with the minimum cost. The source of supply disruption could be related to the manufacturing process or the market. It is assumed in the model used in this study that facilities may be failed for reasons such as equipment failure, shortage of parts and labor shortages. It is also assumed when the distribution center is disturbed, it only misses a part of the capacity and still be able to be in service.

\footnotetext{
* Corresponding author.

E-mail address: atus@pamukkale.edu.tr (H. Angazi)

(C) 2016 Growing Science Ltd. All rights reserved.

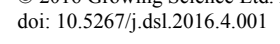


The model in this study also considers the partial backorder status for the shortcoming. Many studies with existing systems generally focused on the following two conditions during the lack of inventory in the existing system (e.g. Huang et al., 2006): it is probable that total demand be faced with delay in the delivery during the period of shortage or the total demand be lost forever during this period. While, in many real inventory systems it is more reasonable that during the periods of shortage, only part of the demand be considered as a delayed order and the remainder be lost. The inventory models which consider a combination of backorder and shortage resulted from the missed sales, not only are closer to reality but also they could influence the costs of the system (Baita, 1998; Adelman, 2004). In this paper, a combination of two types of shortages is considered including backorder shortage and the shortage resulted from the lost sales and the shortage which is considered as a partial backordering in the problem. Under these conditions, customers who are faced with shortage, decide to whether receive products with delay or withdraw the purchase.

\section{Literature Review}

The distribution network is one of the major factors that have a salient impact on supply chain efficiency. This network is designed to achieve the diverse objectives of the supply chain, which could be related to minimization of network costs or increased accountability. Designing an efficient distribution network includes three major decisions: locating facilities, vehicle routing and inventory control. Due to the high dependence of these decisions, in general, in the supply chain literature, many researchers have tried to consider these decisions together, these studies have led to the emergence of issues such as: location-routing, routing-inventory and location-inventory (Min et al., 1998; Nagy \& Salhi, 2007). Many studies have been performed on the routing issues (e.g., Baita et al., 1998; Jaillet et al., 2002; Kleywegt et al., 2002; Adelman, 2004; Gaur \& Fisher, 2004; Zhao et al., 2008; Yu et al., 2008). Also studies have been done on the problem of inventory location. But considering simultaneously all three decisions of location, inventory control and routing have attracted attention of few researchers. Shen and Qi (2007) changed the model proposed by Daskin et al. (2002) and imported the estimation of routing costs into the model. These estimates are intended solely dependent on the location of distribution centers. They showed that with regard to the cost of routing, it is possible to significantly reduce network costs. But their proposed model only has considered the decisions related to location and inventory control in terms of decision variables.

Liu and Lee (2003) studied three decisions of location, routing and inventory control by considering the issue in terms of vehicle routing problem in supply chain design. The model considered a location routing problem with several depots to determine the optimal location of depots. They expressed that although the majority of location problems do not consider inventory decisions, however these decisions are dependent on each other. Therefore, they added the inventory decisions to the locationrouting model. In their model, the demand is probable and the shortage was allowed. In order to achieve the optimal solution, they developed a mathematical programming model but since this was an NPHard problem, the mathematical model cannot be solved effectively. Thus, they proposed a two-stage heuristic algorithm for solving this problem.

Liu and Lin (2005) examined the proposed problem by Liu and Lee (2003). They stated that several heuristic algorithms could be proposed to solve this problem, however, these algorithms are generally trapped in a local optimum solution. So they divided it into two problems of allocation location and inventory routing in order to solve this problem. Next, they proposed a heuristic algorithm resulted from combining Tabu Search algorithm and the Simulated Annealing to solve the problem. The computational results showed that their proposed heuristic algorithm was better than existing methods. Max and Qi, 2007) studied a problem similar to this problem. They considered a supply chain network design problem that must determine the number and location of distribution centers. The customers have a potential demand and each distribution center maintains a certain level of ensured inventory in order to have a specified level of guaranteed service. The intended objective function which was 
considered by them includes the minimization of total costs including the launching costs of distribution centers and inventory maintenance and an estimate of the costs of transportation. They formulate this problem as a nonlinear integer programming model. Then they used a solution algorithm based on Lagrange Release to solve this problem. Their computational experiments include problems with 40 to 320 customers. The computational results show that integration of location and inventory decisions and taking into account transport costs had an advantage over the other models.

Zhang et al., (2008) studied the problem of location-routing- inventory in a multi- period multi-depot status. They considered a single-product model where each path should be closely traveled by a vehicle and each path starts from the depot and ends to the depot. In their studied problem, the transportation system is assumed to be homogeneous, it is also assumed that the customer demand follows a Poisson distribution. Since their problem belongs to the category of NP-Hard problems, the mathematical model cannot be solved effectively. So they proposed a hybrid genetic algorithm to efficiently solve the problem. Ahmadi Javid and Azad (2010) studied the decisions of locating facilities, routing and inventory control in a supply chain network design, for the first time. The demand was likely in their proposed model and each distribution center holds a confidence level of inventory to maintain a certain level of service. They assumed that each distribution center follows an inventory policy $(\mathrm{Q}, \mathrm{R})$, so that when the level of inventory in the distribution center is less than $R$, the amount of $Q$ units are ordered. They presented a convex integer programming model to achieve the optimal solution of this problem. Since their studied problem is in the category of NP-Hard problems, the mathematical model cannot efficiently solve it. Therefore, they proposed a heuristic method based on Tabu Search and Simulated Annealing to efficiently solve the problem. Computational results show the effectiveness of the proposed algorithm. Xuefeng (2010) studied an integrated location inventory routing problem for a chain organization. The distribution system which was studied by him consists of a central warehouse and some distribution centers and retailers. Retailers have the potential demand and receive services by the distribution centers in the same period of demand. The objective was minimizing the cost of the system including the cost of setting up distribution centers and transportation costs. He developed a mathematical programming model to optimally solve the problem. Then he offered a solution algorithm based on the nested releasing method to efficiently solve the problem. His computational experiments include solving problems with 50 to 150 customers. The computational results show the efficiency of the proposed solution

Tavakkoli-Moghaddam et al. (2013) studied a three-level supply chain network design problem with single-source. For the first time, they proposed a new mathematical model considering the risk pooling, the decision related to the inventory on the location of distribution centers, potential demand, existence of different methods of transport and vehicle routing from distribution centers to customers. They formulated this in the form of a two-objective nonlinear mathematical programming model. The aim of their proposed model was to determine the location and the capacity of distribution centers and allocate them to customers and decisions related to inventory control and vehicle routing. They assumed that the vehicle had to start its path from a distribution center and return to the same center. They developed the model using the software Lingo and then solve it.

In this study the decisions of location, routing and inventory control are considered interrogational in design of the supply chain network. Uncertainties in the studied models are the potential demand and disruption risks. Considering the disruption risks has not been studied for the problem of locationrouting-inventory in the supply chain. The proposed issue is explained in the next section and a mathematical programming model will be developed for this problem. In this study, the three decisions of location, routing and inventory control are considered in a comprehensive model under uncertainty of the disruption risks. 


\section{The deterministic model for supply network design}

In this study, the deterministic model is explained for supply network design and then its likely status and uncertainty will be offered..

\section{Assumptions}

- The issue is investigated in a single-period and single-product status.

- Customers demand is definite.

- The shortage is permitted.

- Each distribution center will receive direct orders from the factory.

- Each customer receives service just from one vehicle.

- The fleet is similar and the capacity is limited.

- Each route starts from a distribution center and ends to the same center.

- Each of potential distribution centers has an integrated capacity that one of the objectives is to find the optimal capacity of each distribution centers for activation. There are a certain fixed launching cost to open a distribution center with a certain capacity.

- A fixed cost is considered for ordering and a cost is intended for the inventory maintenance in each distribution center

- The complementary inventory policy for each distribution center, $(r, Q)$ is given the lack of partial backorder. When the level of inventory in the $j$ distribution center reaches the reorder point of $r_{j}$, a constant value of $Q j$ is ordered to the supplier. Also, each distribution center makes an inventory stores to avoid shortages during the delivery order.

- Each client demand is supplied only through a distribution center.

Indexes and parameters

$\mathrm{k}=\{1, \ldots, \mathrm{B}\}$ total customers

$\mathrm{J}=\{1, \ldots, \mathrm{J}\}$ total distribution centers

$\mathrm{V}$ total routes to distribution center of $\mathrm{j}$

$H=\{k \cup J\}$ a set of integration of total customers and potential distribution centers

$\mu_{k}$ The annual demand of the $\mathrm{k}$-th customer

$f_{j}$ cost of setup of distribution center $\mathrm{j}$

$\lambda_{j}$ Per-unit capacity setup cost for $\mathrm{DC}_{\mathrm{j}}$

$a_{j} \quad$ fixed cost per order placed to the supplier by $\mathrm{DC}_{\mathrm{j}}$

$h_{j}$ The annual inventory holding cost per unit of product in $\mathrm{DC}_{\mathrm{j}}$

$g_{j}$ Fixed cost per shipment from the supplier to the $\mathrm{DC}_{\mathrm{j}}$

$b_{j}$ per-unit shipment cost from the supplier to the $\mathrm{DC}_{\mathrm{j}}$

$d_{k l}$ Transportation cost unit between the two nodes $\mathrm{k}$ and $\mathrm{l}(\forall k, l \in H)$

$L_{j}$ lead time of distribution center $\mathrm{j}$ in year

$\pi_{j}$ Lost sales cost per unit product for $\mathrm{DC}_{\mathrm{j}}$

$\pi_{j}^{\prime}$ fixed penalty cost per unit short in $\mathrm{DC}_{\mathrm{j}}$

cap $_{j}$ Maximum capacity available for distribution center $\mathrm{j}$

$v c$ The annual delivery capacity of a vehicle

$m$ A vehicle commuting times during the year

$Z_{p}$ Level of service ( $p$ percentage of customer's orders that are required to meet) 
$\alpha$ A percentage of demand that is not satisfied and will become backorder

$\theta$ Weighting factor associated with inventory costs

$\beta$ Weighting factor associated with the transportation costs of decision making variables

\section{Decision making variables}

$x_{k p v}$ is equal to 1 if vehicle $V$ passes its customer $k$ in its p-th route

$u_{j v}$ is equal to 1 if the vehicle $v$ be assigned to the depot $j$

$y_{k v}$ is equal to 1 if the customer $k$ is the latest customer of vehicle $V$.

$W_{j} \quad$ If the distribution center $j$ be activated it equals to 1 and otherwise takes a value of zero.

$Y_{j k} \quad$ If the customer $k$ be assigned to the distribution center $j$ it equals to 1 and otherwise takes a value of zero.

$R_{k l v} \quad$ If the node $k$ on the node 1 be prior in the route of the vehicle $V$, it equals to 1 and otherwise takes a value of zero.

$Q_{j} \quad$ The optimal order quantity in the distribution center $j$

$H_{k v} \quad$ Auxiliary variable for customer $k$, in order to vehicle $V$ not become sub-tour in the route.

\section{Cost components of the objective function}

The launching cost of distribution centers

$$
\sum_{j \in J}\left(W_{j} f_{j}+\lambda_{j} \sum_{k \in K} \mu_{k} Y_{j k}\right)
$$

The annual cost for routing from activate distribution centers to customers

$\beta m \sum_{v \in V} \sum_{i \in H} \sum_{l \in J} d_{k l} R_{k l v}$

Direct transportation costs include transportation from supplier to the distribution center

$\beta \sum_{j \in J}\left(g_{j}+b_{j} Q_{j}\right) \frac{\sum_{k \in K} \mu_{k} Y_{j k}}{Q_{j}}$.

Expected number of outstanding payments in each period of $\alpha B(r)$, and the amount of lost profits in each period of $(1-\alpha) B(r)$ where $(0 \leq \alpha \leq 1) \quad \alpha$ is a part of the demand that will be delayed during the shortage period, since the annual cost of shortage is $\frac{D}{Q}\left[\pi_{j}+\pi_{j}^{\prime}(1-\alpha)\right] B(r)$. The expected net inventory level before delivery of order $(1-\alpha) B(r)$ and the expected net inventory level at the beginning of the period $Q+(1-\alpha) B(r)$. Thus, the annual maintenance cost is equal to $h\left[\frac{Q}{2}+(1-\alpha) B(r)\right]$. Hence, the annual inventory cost including ordering and inventory maintenance costs and the shortage will be as follows:

$\sum_{j} a_{j} \frac{\sum_{k \in K} \mu_{k} Y_{j k}}{Q_{j}}+\sum_{j} \sum_{s} q_{s}\left(\frac{\left(1-r_{s j}\right) Q_{j}}{2} \theta h_{j}+(1-\alpha) r_{s j} Q_{j} \theta h_{j}\right)+$
$\sum_{j} \sum_{s} q_{s}\left[\pi_{j}+\pi_{j}^{\prime}(1-\alpha)\right] \sum_{k \in K}\left(1-P_{j k s}\right) \mu_{k}$ 
The problem is formulated as follows according to the assumptions, parameters and cost components of the objective function

$$
\begin{aligned}
& \min : \sum_{j \in J}\left(W_{j} f_{j}+\lambda_{j} \sum_{k \in K} \mu_{k} Y_{j k}\right)+\beta m \sum_{v \in V} \sum_{i \in H} \sum_{l \in J} d_{k l} R_{k l v} \\
& \quad+\sum_{j \in J}\left(\left(\alpha_{j}+\beta g_{j}\right) \frac{\sum_{k \in K} \mu_{k} Y_{j k}}{Q_{j}}+\beta b_{j} \sum_{k \in K} \mu_{k} Y_{j k}\right) \\
& \sum_{p} \sum_{v} x_{k p v}=1 \quad \forall k \\
& \sum_{k} x_{k p v} \leq 1 \quad \forall p, v \\
& \sum_{v} u_{j v} \leq M \times W_{j} \forall j \\
& \sum_{j} u_{j v} \leq 1 \quad \forall v \\
& x_{k p v}-\sum_{k} x_{k, p+1, v} \leq y_{k v} \forall p, k, v \\
& \sum_{k} x_{k, p+1, v} \leq \sum_{k} x_{k, p, v} \forall v, p \\
& Y_{j k} \geq u_{j v}+\sum_{p} x_{k, p, v}-1 \quad \forall j, k, v \\
& \sum_{k \in K} \mu_{k} \sum_{p} x_{k, p, v} \leq V C \quad \forall v \in V \\
& \sum_{k \in K} \mu_{k} Y_{j k} \leq W_{j} c a p_{j} \quad \forall j \\
& x_{k, p, v}+x_{l, p+1, v} \leq 1+R_{k l v} \forall k, l, v, p \\
& u_{j v}+x_{l, 1, v} \leq 1+R_{j l v} \forall j, l, v \\
& u_{j v}+y_{k v} \leq 1+R_{k j v} \forall j, k, v \\
& Y_{j k}, W_{j}, R_{k l v}, x_{k p v}, u_{j v}, y_{k v} \in\{0,1\} \\
& \forall j \in J
\end{aligned}
$$

In Eq. (1) the expected total cost function including fixed and variable costs of operating distribution centers, the annual cost for routing from activate distribution centers to customers, the annual inventory cost including ordering and inventory maintenance costs and transportation cost from the supplier to distribution center $j$ and the annual cost of maintaining; will be minimized. Constraint (2) expresses that each customer must be assigned exactly to one place in the route 1 of the vehicle. Constraint (3) expresses that finally one customer will be assigned to each route of each vehicle. Constraint (4) expresses that if the distribution center $j$ is not used, no vehicle can be assigned to it.

Constraint (5) expresses that each vehicle can only be assigned to one distribution center. Constraint (6) is applied to calculate the final task in the path of the vehicle $v$. To put it more precisely, if task $k$ be in place $\mathrm{p}$ in the route of this vehicle and there is nothing in the $p+1$ place, the task $k$ will be the last task in the route of vehicle $V$. Constraint (7) expresses that the locations in the path of each vehicle 
should be filled up ascending, namely, the first place must be filled first of all then the second and so on. Constraint (8) is to calculate the allocation of customers to distribution centers. Under this constraint if the task $k$ and the distribution center $j$ both are assigned to a vehicle, the task $k$ is allocated to the distribution center $j$.

Constraint (9) is to ensure that the vehicles' capacities are being complied with. Constraint (10) represents the limitation on capacity of the distribution centers. Constraint (11) is to calculate the variable $R_{k l v}$, which means that if for the vehicle $v$, the tasks $k$ and $l$ be in tandem, $R_{k l v}$ is equal to 1 . Similarly, the Constraints (12) and (13) are to calculate $R_{j l v}$ and $R_{k j v}$ between the first and last job of the vehicle and the distribution center. Constraint (14) shows the binary variables and constraint (15) shows no-negavity variables behavior.

\section{The proposed research model}

In this paper, the design of supply chain network is studied given the random disruptions in facilities and locating distribution centers in a three-level supply chain which includes suppliers, distribution centers and customers (See Fig. 1). The following is an overview of the proposed model.

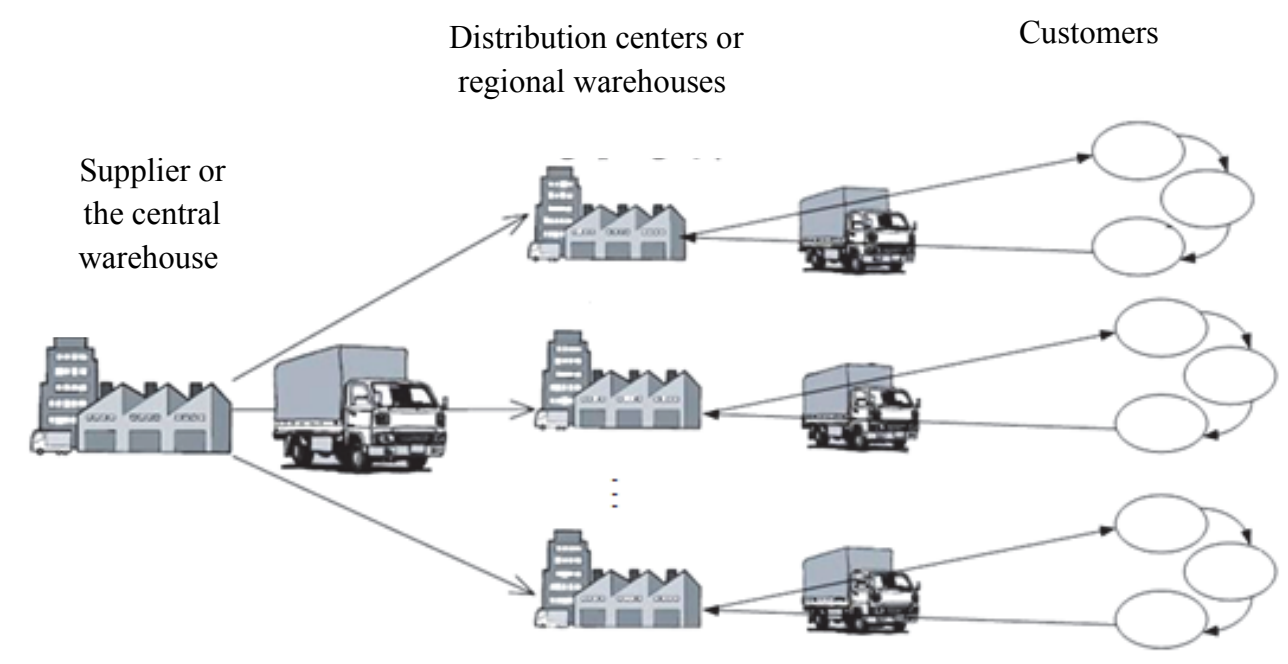

Additional Assumptions

Fig. 1. structure of the model

- Customers' demands are independent from each other and they are normally distributed.

- Depending on the environment, shortage is permitted.

\section{Additional Indexes and parameters}

$S$ the set of failure scenarios

$\mu_{k}$ The means of customers' yearly demand

Cost components of the objective function

The launching cost of distribution centers

$$
\sum_{j \in J}\left(W_{j} f_{j}+\lambda_{j} \sum_{k \in K} \mu_{k} Y_{j k}\right)
$$


The annual cost for routing from activate distribution centers to customers

$$
\beta m \sum_{v \in V} \sum_{i \in H} \sum_{l \in J} d_{k l} R_{k l v}
$$

The annual cost of maintaining confidence inventory

$$
\sigma_{j}^{2}=L_{j} \sum_{k \in K} \sigma_{k}^{2} Y_{j k} \Rightarrow S S=Z_{P} \sqrt{\sigma_{j}^{2}} \Rightarrow \theta h_{j} Z_{P} \sqrt{L_{j} \sum_{k \in K} \sigma_{k}^{2} Y_{j k}}
$$

$\sigma_{j}^{2}$ is the variance of the distribution center $j$ which equals to the sum of the variance of customers who are dedicated to this distribution center multiplied in delivery time of the distribution center $j$. Direct transportation costs include transportation from supplier to the distribution center

$$
\beta \sum_{j \in J}\left(g_{j}+b_{j} Q_{j}\right) \frac{\sum_{k \in K} \mu_{k} Y_{j k}}{Q_{j}}
$$

Expected number of outstanding payments in each period of $\alpha B(r)$, and the amount of lost profits in each period of $(1-\alpha) B(r)$ where $(0 \leq \alpha \leq 1) \quad \alpha$ is a part of the demand that will be delayed during the shortage period, since the annual cost of shortage is $\frac{D}{Q}\left[\pi_{j}+\pi_{j}^{\prime}(1-\alpha)\right] B(r)$.

The expected net inventory level before delivery of order $(1-\alpha) B(r)$ and the expected net inventory level at the beginning of the period is $Q+(1-\alpha) B(r)$. Thus, the annual maintenance cost is equal to $h\left[\frac{Q}{2}+(1-\alpha) B(r)\right]$. Hence, the annual inventory cost including ordering and inventory maintenance costs and the shortage will be as follows:

$$
\sum_{j} a_{j} \frac{\sum_{k \in K} \mu_{k} Y_{j k}}{Q_{j}}+\sum_{j} \sum_{s} q_{s}\left(\frac{\left(1-r_{s j}\right) Q_{j}}{2} \theta h_{j}+(1-\alpha) r_{s j} Q_{j} \theta h_{j}\right)+\sum_{j} \sum_{s} q_{s}\left[\pi_{j}+\pi_{j}^{\prime}(1-\alpha)\right] \sum_{k \in K}\left(1-P_{j k s}\right) \mu_{k}
$$

The problem is formulated as follows according to the assumptions, parameters and cost components of the objective function

$$
\begin{aligned}
& \min : \sum_{j \in J}\left(W_{j} f_{j}\right.\left.+\lambda_{j} \sum_{k \in K} \mu_{k} Y_{j k}\right)+\beta m \sum_{v \in V} \sum_{i \in H} \sum_{l \in J} d_{k l} R_{k l v}+\sum_{j \in J}\left(\left(\alpha_{j}+\beta g_{j}\right) \frac{\sum_{k \in K} \mu_{k} Y_{j k}}{Q_{j}}+\beta b_{j} \sum_{k \in K} \mu_{k} Y_{j k}\right) \\
&+\sum_{j \in J}\left(\sum_{s} q_{s}\left(\frac{\left(1-r_{s j}\right) Q_{j}}{2} \theta h_{j}+\theta h_{j}(1-\alpha) r_{s j} Q_{j}\right)+\theta h_{j} Z_{p} \sqrt{L_{j} \sum_{k \in K} \sigma_{k}^{2} Y_{j k}}\right) \\
&+\sum_{j \in J} \sum_{s} q_{s}\left(\pi_{j}+\dot{\pi}_{j}(1-\alpha)\right) \sum_{k \in K}\left(1-P_{j k s}\right) \mu_{k} \\
& \sum_{p} \sum_{v} x_{k p v}=1 \quad \forall k \\
& \sum_{k}^{k} x_{k p v} \leq 1 \quad \forall p, v \\
& \sum_{v} u_{j v} \leq M \times W_{j} \quad \forall j \\
& \sum_{j} u_{j v} \leq 1 \quad \forall v \\
& x_{k p v}-\sum_{k} x_{k, p+1, v} \leq y_{k v} \forall p, k, v
\end{aligned}
$$




$$
\begin{aligned}
& \sum_{k} x_{k, p+1, v} \leq \sum_{k} x_{k, p, v} \forall v, p \\
& Y_{j k} \geq u_{j v}+\sum_{p} x_{k, p, v}-1 \quad \forall j, k, v \\
& \sum_{k \in K} \mu_{k} \sum_{p} x_{k, p, v} \leq V C \quad \forall v \in V \\
& \sum_{k \in K} \mu_{k} Y_{j k} \leq W_{j} c a p_{j} \quad \forall j \\
& \sum_{k \in K} P_{j k s} \mu_{k} \leq\left(1-r_{s j}\right) c a p_{j} \quad \forall j, s \\
& P_{j k s} \leq Y_{j k} \quad \forall j, k, s \\
& x_{k, p, v}+x_{l, p+1, v} \leq 1+R_{k l v} \forall k, l, v, p \\
& u_{j v}+x_{l, 1, v} \leq 1+R_{j l v} \forall j, l, v \\
& u_{j v}+y_{k v} \leq 1+R_{k j v} \forall j, k, v \\
& Y_{j k}, W_{j}, R_{k l v}, x_{k p v}, u_{j v}, y_{k v} \in\{0,1\} \\
& Q_{j}>0 \quad \forall j \in J \\
& P_{j k s} \geq 0 \quad \forall j \in J, k \in K, s \in S
\end{aligned}
$$

Most equations are the same as the previous mathematical model. In the event of a risk, the capacity of distribution centers decreases therefore, it is not possible to meet all customer demands and for each client $k$ under scenario $s$ the amount of $P_{j k s} \%$ of the demand is met, the total amount of customer demands should not exceed the capacity of the distribution center. Constraint (26) indicates that if the distribution $j$ is not assigned to the customer $k$, then it is not possible to use this distribution center the meet the customer $k$ demand.

\section{Solution}

In this part, a solution strategy is presented to solve the problem described in the previous section based on the Outer Approximation Method. First, the Outer Approximation Method is explained then this algorithm will be applied to solve the proposed problem. The Outer Approximation is an efficient method for solving nonlinear integer programming problems presented by Duran and Grossmann (1986) to solve certain types of problems in nonlinear integer programming. They assumed that variables are either integer or linear and nonlinear functions are convex. Then, they proposed an efficient algorithm to solve this kind of problem in nonlinear integer programming based on analysis, Outer Approximation and release. Their proposed Outer Approximation Algorithm iteratively solves a problem in integer mixed linear programming and a sub-problem in non-linear programming (no integers) converges to the optimal solution with a predetermined approximation. They implemented their proposed algorithm for several examples and analyzed them. The algorithm is considered as the first outer approximation algorithm and has some major limitations: first, in terms of integer variables, it assumed that the problem is linear. This assumption limits the application of their proposed algorithm for solving problems in nonlinear integer programming. In addition, their proposed algorithm has not provided a detailed procedures for how to deal with infeasibility of the sub-problem. To overcome this limitation Fletcher and Leyffer (1994) developed the outer approximation algorithm for solving problems in nonlinear integer programming, with no assumption of linearity relative to integer variable and provided a detailed procedures for how to deal with infeasibility of the sub-problem. To solve the proposed problem through the outer approximation method, first, the intended problem should become convex. In the intended issue, just the objective function has nonlinear functions. In better words, the objective function has two nonlinear functions. The first function is $\sum_{j \in J}\left(\left(\alpha_{j}+\right.\right.$ 
$\left.\left.\beta g_{j}\right) \frac{\sum_{k \in K} \mu_{k} Y_{j k}}{Q_{j}}\right)$ which is non-linear with respect to the variable $Q_{j}$. This function is a convex function and meets the initial condition of using the outer approximation algorithm. However, the second function $\sum_{j \in J}\left(\theta h_{j} Z_{p} \sqrt{L_{j} \sum_{k \in K} \sigma_{k}^{2} Y_{j k}}\right)$ which is non-linear with respect to the variable $Y_{j k}$ is not convex in the present form. There are binary variables to make this function convex: $\mathrm{Y}_{\mathrm{jk}}^{2}=$ $\mathrm{Y}_{\mathrm{jk}}$. Therefore, the objective function becomes convex in the form below:

$$
\begin{aligned}
\min : \sum_{j \in J}\left(W_{j} f_{j}+\right. & \left.\lambda_{j} \sum_{k \in K} \mu_{k} Y_{j k}\right)+\beta m \sum_{v \in V} \sum_{i \in H} \sum_{l \in J} d_{k l} R_{k l v}+\sum_{j \in J}\left(\left(\alpha_{j}+\beta g_{j}\right) \frac{\sum_{k \in K} \mu_{k} Y_{j k}}{Q_{j}}+\beta b_{j} \sum_{k \in K} \mu_{k} Y_{j k}\right) \\
& +\sum_{j \in J}\left(\sum_{s} q_{s}\left(\frac{\left(1-r_{s j}\right) Q_{j}}{2} \theta h_{j}+\theta h_{j}(1-\alpha) r_{s j} Q_{j}\right)+\theta h_{j} Z_{p} \sqrt{L_{j} \sum_{k \in K} \sigma_{k}^{2} Y_{j k}^{2}}\right) \\
& +\sum_{j \in J} \sum_{s} q_{s}\left(\pi_{j}+\dot{\pi}_{j}(1-\alpha)\right) \sum_{k \in K}\left(1-P_{j k s}\right) \mu_{k}
\end{aligned}
$$

Now, to solve the problem by the outer approximation method the sob-problem will be developed as following:

$$
\begin{aligned}
& \operatorname{min:} \sum_{j \in J}\left(\widehat{W_{J}} f_{j}+\right.\left.\lambda_{j} \sum_{k \in K} \mu_{k} \widehat{Y_{j k}}\right)+\beta m \sum_{v \in V} \sum_{i \in H} \sum_{l \in J} d_{k l} \widehat{R_{k l v}} \\
&+\sum_{j \in J}\left(\left(\alpha_{j}+\beta g_{j}\right) \frac{\sum_{k \in K} \mu_{k} \widehat{Y_{J k}}}{Q_{j}}+\beta b_{j} \sum_{k \in K} \mu_{k} \widehat{Y_{J k}}\right) \\
&+\sum_{j \in J}\left(\sum_{s} q_{s}\left(\frac{\left(1-r_{s j}\right) Q_{j}}{2} \theta h_{j}+\theta h_{j}(1-\alpha) r_{s j} Q_{j}\right)+\theta h_{j} Z_{p} \sqrt{L_{j} \sum_{k \in K} \sigma_{k}^{2} \widehat{Y_{J k}^{2}}}\right) \\
&+\sum_{j \in J} \sum_{s} q_{s}\left(\pi_{j}+\dot{\pi}_{j}(1-\alpha)\right) \sum_{k \in K}\left(1-P_{k s}\right) \mu_{k} \\
& \sum_{k \in K} P_{j k s} \mu_{k}-\left(1-r_{s j}\right) c a p_{j} \leq 0 \quad \forall j, s \\
& P_{j k s} \leq \widehat{Y_{j k}} \forall \forall j, k, s \\
& P_{j k s}-1 \leq 0 \quad \forall k, s \\
& P_{j k s} \geq 0 \\
& Q_{j}>0
\end{aligned}
$$

This sub-problem is easily obtained through the assumption that the integer variables are constant. In this model, the hat sign on the variable indicates that they are constant. Therefore, the sub-problem is a non-linear and without integer variables. If the sub-problem be infeasible, the amount of infeasibility will be minimized and the minimization of the amount of infeasibility will be performed as follows:

$$
\begin{aligned}
& \min : \sum_{j \in J} \sum_{s \in S} g_{1}(j, s)+\sum_{j \in J} \sum_{k \in K} \sum_{s \in S} g_{2}(j, k, s)+\sum_{k \in K} \sum_{s \in S} g_{3}(k, s)(1) \\
& \sum_{k \in K} P_{j k s} \mu_{k}-\left(1-r_{s j}\right) c a p_{j} \leq g_{1}(j, s) \quad \forall j, s \\
& P_{j k s} \leq \widehat{Y_{j k}}+g_{2}(j, k, s) \forall j, k, s \\
& P_{j k s}-1 \leq g_{3}(k, s) \quad \forall k, s \\
& P_{j k s} \geq 0 \\
& Q_{j}>0
\end{aligned}
$$

The OA Master problem is developed as follows:

min: $\eta$

$\eta \leq U B D-\epsilon$ 


$$
\begin{aligned}
& \eta \geq \sum_{j \in J}\left(W_{j} f_{j}+\lambda_{j} \sum_{k \in K} \mu_{k} Y_{j k}\right)+\beta m \sum_{v \in V} \sum_{i \in H} \sum_{l \in J} d_{k l} R_{k l v} \\
& +\sum_{j \in J}\left(\left(\alpha_{j}+\beta g_{j}\right) \frac{\sum_{k \in K} \mu_{k} \widehat{Y_{j k}^{h}}}{\widehat{Q_{J}^{h}}}+\beta b_{j} \sum_{k \in K} \mu_{k} Y_{j k}\right) \\
& +\sum_{j \in J}\left(\sum_{s} q_{s}\left(\frac{\left(1-r_{s j}\right) Q_{j}}{2} \theta h_{j}+\theta h_{j}(1-\alpha) r_{s j} Q_{j}\right)+\theta h_{j} Z_{p} \sqrt{L_{j} \sum_{k \in K} \sigma_{k}^{2} \widehat{\left(Y_{j k}^{h}\right)^{2}}}\right) \\
& +\sum_{j \in J} \sum_{s} q_{s}\left(\pi_{j}+\dot{\pi}_{j}(1-\alpha)\right) \sum_{k \in K}\left(1-P_{j k s}\right) \mu_{k} \\
& +\sum_{j \in J}\left(\left(\alpha_{j}+\beta g_{j}\right) \frac{\sum_{k \in K} \mu_{k}}{\widehat{Q_{J}^{h}}}\left(Y_{j k}-\widehat{Y_{J k}^{h}}\right)\right) \\
& +\sum_{j \in J}\left(\left(\alpha_{j}+\beta g_{j}\right) \frac{-\sum_{k \in K} \mu_{k} \widehat{Y_{J k}}}{{\widehat{Q_{J}^{h}}}^{2}}\left(Q_{j}-\widehat{Q_{J}^{h}}\right)\right) \\
& +\sum_{j \in J}\left(\theta h_{j} Z_{p} \frac{L_{j} \sum_{k \in K} \sigma_{k}^{2}\left(\widehat{Y_{j k}^{h}}\right)\left(Y_{j k}-\widehat{Y_{j k}^{h}}\right)}{\sqrt{L_{j} \sum_{k \in K} \sigma_{k}^{2} \widehat{Y_{j k}^{2}}}}\right) \quad \forall h \in H \\
& \sum_{p} \sum_{v} x_{k p v}=1 \quad \forall k \\
& \sum x_{k p v} \leq 1 \quad \forall p, v \\
& \sum_{i}^{k} u_{j v} \leq M \times W_{j} \quad \forall j \\
& \sum_{j}^{v} u_{j v} \leq 1 \quad \forall v \\
& x_{k p v}-\sum_{k} x_{k, p+1, v} \leq y_{k v} \forall p, k, v \\
& \sum_{k} x_{k, p+1, v} \leq \sum_{k} x_{k, p, v} \forall v, p \\
& Y_{j k} \geq u_{j v}+\sum_{p} x_{k, p, v}-1 \quad \forall j, k, v \\
& \sum_{k \in K} \mu_{k} \sum_{p} x_{k, p, v} \leq V C \quad \forall v \in V \\
& \sum_{k \in K} \mu_{k} Y_{j k} \leq W_{j} \operatorname{cap}_{j} \quad \forall j \\
& \sum_{k \in K}\left(P_{j k s}\right) \mu_{k} \leq\left(1-r_{s j}\right) \operatorname{cap}_{j} \quad \forall j, s \\
& P_{j k s} \leq Y_{j k} \quad \forall j, k, s \\
& x_{k, p, v}+x_{l, p+1, v} \leq 1+R_{k l v} \forall k, l, v, p \\
& u_{j v}+x_{l, 1, v} \leq 1+R_{j l v} \forall j, l, v \\
& u_{j v}+y_{k v} \leq 1+R_{k j v} \forall j, k, v
\end{aligned}
$$

In this model, $H$ is the set of iterations. $\varepsilon$ is a small number which will determine the accuracy of the algorithm. In other words (if the sub-problem is resolved to the global optimality) it is proved that the algorithm reaches an optimal solution $-\in$ (Fletcher and Leyffer, 1994). The $-\varepsilon$ is an optimal answer that has the maximum error $\varepsilon \times(\mathrm{opt})$ where opt is the optimal solution. More precisely, if the sub-problem is resolved to the global optimality, it will be ensured that: $O A_{\text {sol }} \leq o p t \times(1+\varepsilon)$. In this equation $O A_{\text {sol }}$ is the final answer of the outer approximation algorithm. The general trend of the proposed outer approximation algorithm is listed below: 
Initialize: $W_{j}^{0}, Y_{j k}^{0}, R_{k l v^{\prime}}^{0}:$ set $l=0, H^{-1}=\varnothing$, and $U B D=\infty$

Repeat

(1) Solve sub-problem given in Eqs. (34-39) or the feasibility problem given by Eqs. (40-45) if the first sub-problem is infeasible let the solution be $\left(Q_{j}^{l}, P_{k s}^{l}\right)$.

(2) Linearize the nonlinear constraint around the point $\left(W_{j}^{l}, Y_{j k}^{l}, R_{k l v}^{l}, Q_{j}^{l}, P_{k s}^{l}\right)$ and set $H^{l}=H^{l-1} \cup(l)$

(3) If the first sub-problem is feasible and $f^{l}\left(W_{j}^{l}, Y_{j k}^{l}, R_{k l v^{\prime}}^{l}, Q_{j}^{l}, P_{k s}^{l}\right)<U B D$ then: Update the current best solution $\left(W_{j}^{*}, Y_{j k}^{*}, R_{k l v^{\prime}}^{*}, Q_{j}^{*}, P_{k s}^{*}\right)$ and $U B D$.

(4) Solve the first sub-problem to obtain $\left(W_{j}^{l+1}, Y_{j k}^{l+1}, R_{k l v^{\prime}}^{l+1}\right)$. Set $l=l+1$

Until the second sub-problem is infeasible.

Fig. 2. The pseudo-code of the outer approximation algorithm

According to this pseudo-code, first, an initial value is obtained for the integer variables. This initial value is acquired through the solution of the model presented in section 3, by eliminating the non-linear sentences, in the form of the linear mixed integer programming model. After obtaining the constant for integer variables, sub-problems given by Eq. (34) to Eq. (39) are formed and solved. If the sub-problem is feasible and the obtained solution be lower than the best solution obtained so far (UBD), this value will be updated. If the sub-problem becomes infeasible, by solving the models Eq. (40) to Eq. (45) the infeasibility will be minimized.

In both cases the values of $\left(Q_{j}^{l}, P_{k S}^{l}\right)$ are obtained and these values with pre-obtained integer variables will be used to outer approximation and linearization of the objective function (a new constraint is added to the original problem). By adding a new constraint to the original problem, the master problem is updated and solved in order to find new values for the integer variables. The new integer values are used again as constants for the formation of the sub-problem. Then again, the sub-problem is solved to obtain new values of $\left(Q_{j}^{l}, P_{k s}^{l}\right)$ in order to add a new constraint to the problem through these values and the fixed values of integer variables. This is repeated until the master problem won't be infeasible. After the end of the algorithm, UBD is the value of the optimal objective function and $\left(W_{j}^{*}, Y_{j k}^{*}, R_{k l v}^{*}, Q_{j}^{*}, P_{j k s}^{*}\right)$ is the optimal solution.

\section{Computational results}

In this section several examples with diverse measures were solved to compare the performance of the proposed outer approximation algorithm with the proposed nonlinear integer programming model. Also the performance of the outer approximation algorithm were studied in more detail and managerial analyses were performed. The nonlinear integer programming model and the proposed outer approximation algorithm both were implemented in the software GAMS 24.1.2. The computer that was used to perform the experiments is a personal computer with the following characteristics:

Intel core i3 processor $2.4 \mathrm{GHz}$ and $4 \mathrm{~GB}$ of RAM

To solve MINLP, MILP and NLP problems, the solvers BINMIN, CPLEX and CONOPT are used in the medium of the software Gomez, respectively. The generated examples were produced to implement the computational experiments in a random way as Table 1. 


\section{Table 1}

How the random examples are generated

\begin{tabular}{|c|c|}
\hline Parameter & Value \\
\hline The means of customers'yearly demands $\left(\mu_{k}\right)$ & Uniform (400-1500) \\
\hline variances of yearly demand of customer $\left(\sigma_{k}^{2}\right)$ & Uniform (10-30) \\
\hline cost of setup of distribution center $\mathrm{j}\left(f_{j}\right)$ & $0.9 * \mathrm{k}_{\mathrm{j}}\left(\mathrm{k}_{\mathrm{j}}=\operatorname{Rand}[300,450]\right)$ \\
\hline per-unit capacity setup cost for $\mathrm{DC}_{\mathrm{j}}\left(\lambda_{j}\right)$ & Uniform $(5-15)$ \\
\hline fixed cost per order placed to the supplier by $\mathrm{DC}_{\mathrm{j}}\left(a_{j}\right)$ & Uniform (10-15) \\
\hline The annual inventory holding cost per unit of product in $\mathrm{DC}_{\mathrm{j}}\left(h_{j}\right)$ & Uniform $(5-10)$ \\
\hline Fixed cost per shipment from the supplier to the $\mathrm{DC}_{\mathrm{j}}\left(g_{j}\right)$ & Uniform (10-15) \\
\hline per-unit shipment cost from the supplier to the $\mathrm{DC}_{\mathrm{j}}\left(b_{j}\right)$ & Uniform $(5-10)$ \\
\hline Transportation cost unit between the two nodes $\left(d_{k l}\right)$ & Uniform $(0-300)$ \\
\hline lead time of distribution center $\mathrm{j}$ in year $\left(L_{j}\right)$ & Uniform $\left(\frac{6}{365}, \frac{10}{365}\right)$ \\
\hline Maximum capacity available for distribution center $\mathrm{j}\left(\operatorname{cap}_{j}\right)$ & $\begin{array}{l}2 * C_{j} *[D /|J|] \\
\left(\mathrm{C}_{\mathrm{j}}=\operatorname{Rand}[0.8,1.2]\right)\end{array}$ \\
\hline Lost sales cost per unit product for $\mathrm{DC}_{\mathrm{j}}\left(\pi_{j}\right)$ & Uniform (500-1000) \\
\hline fixed penalty cost per unit short in $\mathrm{DC}_{\mathrm{j}}\left(\pi_{j}^{\prime}\right)$ & Uniform (100-300) \\
\hline The annual delivery capacity of a vehicle $(v c)$ & $2 *[D / V \mid]$ \\
\hline probability that scenario s occurs $\left(q_{s}\right)$ & $\mathrm{S}_{1}=0.6, \mathrm{~S}_{2}=0.4, \mathrm{~S}_{3}=0.2$ \\
\hline percentage of supply at $\mathrm{DC}_{\mathrm{j}}$ which is disrupted in scenario $\mathrm{s}\left(r_{s j}\right)$ & $\begin{array}{l}r_{s_{1} j}=\operatorname{Uniform}(0.05,0.15) \\
r_{s_{2} j}=\operatorname{Uniform}(0.2,0.3) \\
r_{s_{3} j}=\operatorname{Uniform}(0.35,0.5)\end{array}$ \\
\hline fraction of the demand during the stockout period will be backordered $(\alpha)$ & $0 \leq \alpha \leq 1$ \\
\hline
\end{tabular}

\section{Comparison of the outer approximation algorithm and the mathematical model}

Several examples have been solved by the outer approximation model and the proposed model for comparison of the outer approximation algorithm and the mathematical model. For each example, a maximum run time of 2 hours ( 7200 seconds) is considered and the results are given in Table 2. In this table, $J, K$ and $V$ indicate the number of distribution centers, the number of customers and the number of vehicles, respectively. CPU represents the run time and objective represents the obtained value of the objective function. If the mathematical model does not reach the optimal solution in 2 hours, the obtained upper and lower values are reported in the table. In addition, the RPD is calculated using the following formula:

$$
R P D=\frac{U B-L B}{L B} \times 100
$$

In this formula, $L B$ is the lower obtained value through the mathematical model and $U B$ is the upper obtained limit by the mathematical model and/or the outer approximation algorithm. As the lower values of RPD indicate the higher quality of the solution algorithm and the lower values of RPD are bolded in the table. As is clear from Table, the performance of the outer approximation algorithm is much better than the mathematical model. Among 20 examples, in 18 cases the outer approximation 
algorithm obtained batter RPD values and in another two examples that the mathematical model has achieved better RPD values and the difference between the two algorithms was negligible. Also, the run time of the outer approximation algorithm is much less than the solution time of the mathematical model. Thus, the outer approximation algorithm has achieved better results in less time and this represents the efficiency of the outer approximation algorithm.

Table 2

Comparison of the outer approximation algorithms and the mathematical model

\begin{tabular}{|c|c|c|c|c|c|c|c|c|c|}
\hline \multirow{2}{*}{ No. } & \multirow{2}{*}{$\mathrm{J}$} & \multirow{2}{*}{$\mathrm{K}$} & \multirow{2}{*}{$\mathrm{V}$} & \multicolumn{3}{|l|}{ Model } & \multicolumn{3}{|l|}{$\mathrm{OA}$} \\
\hline & & & & CPU (s) & Objective & RPD & CPU (s) & Objective & RPD \\
\hline 1 & 5 & 7 & 3 & 7200 & {$[4733.08,4785.61]$} & 1.11 & 4.16 & 4816.02 & 1.73 \\
\hline 2 & 5 & 7 & 4 & 7200 & {$[18892.37,20136.56]$} & 6.59 & 3.7 & 19922.28 & 5.45 \\
\hline 3 & 5 & 7 & 3 & 7200 & {$[21507.26,21562.49]$} & 0.26 & 8.51 & 21600.23 & 0.43 \\
\hline 4 & 5 & 8 & 4 & 7200 & {$[20936.23,21581.93]$} & 3.08 & 5.07 & 21090.73 & 0.73 \\
\hline 5 & 5 & 8 & 3 & 7200 & {$[19263.67,20841.34]$} & 8.20 & 3.61 & 20234.91 & 5.05 \\
\hline 6 & 5 & 10 & 4 & 7200 & {$[22804.07,23925.48]$} & 4.93 & 5.83 & 23772.41 & 4.25 \\
\hline 7 & 5 & 10 & 3 & 7200 & {$[24262.93,26346.77]$} & 8.60 & 6.79 & 26155.84 & 7.81 \\
\hline 8 & 8 & 10 & 4 & 7200 & {$[17362.35,18524.92]$} & 6.70 & 4.51 & 18240.66 & 5.05 \\
\hline 9 & 8 & 10 & 3 & 7200 & {$[19020.13,21041.70]$} & 10.62 & 4.70 & 19970.85 & 4.99 \\
\hline 10 & 10 & 15 & 4 & 7200 & {$[13775.70,15533.99]$} & 12.76 & 13.95 & 15166.45 & 10.09 \\
\hline 11 & 10 & 15 & 3 & 7200 & {$[22078.19,24431.73]$} & 10.66 & 17.51 & 24132.20 & 9.30 \\
\hline 12 & 10 & 15 & 4 & 7200 & {$[22201.25,25527.49]$} & 14.99 & 14.53 & 24153.29 & 8.80 \\
\hline 13 & 15 & 20 & 3 & 7200 & {$[25448.73,30395.09]$} & 19.44 & 30.8 & 27897.24 & 9.62 \\
\hline 14 & 15 & 20 & 4 & 7200 & [31226.22,35755.57] & 14.50 & 51.11 & 33779.76 & 8.17 \\
\hline 15 & 15 & 25 & 3 & 7200 & {$[40531.16, \infty]$} & $\infty$ & 157.38 & 43217.47 & 6.63 \\
\hline 16 & 15 & 25 & 4 & 7200 & {$[35700.10,40557.90]$} & 13.61 & 69.66 & 38122.37 & 6.78 \\
\hline 17 & 15 & 25 & 3 & 7200 & [34441.30,38630.79] & 12.16 & 156.10 & 36488.93 & 5.94 \\
\hline 18 & 20 & 25 & 4 & 7200 & [29790.30,31535.32] & 5.86 & 40.38 & 31128.44 & 4.49 \\
\hline 19 & 20 & 25 & 3 & 7200 & [26932.69,31405.40] & 16.61 & 56.95 & 29040.53 & 7.82 \\
\hline 20 & 20 & 25 & 4 & 7200 & {$[31344.56,37280.71]$} & 18.94 & 151.7 & 33581.02 & 7.13 \\
\hline
\end{tabular}

For better comparing the two methods, the RPD results are presented in a column chart in Fig. 2.

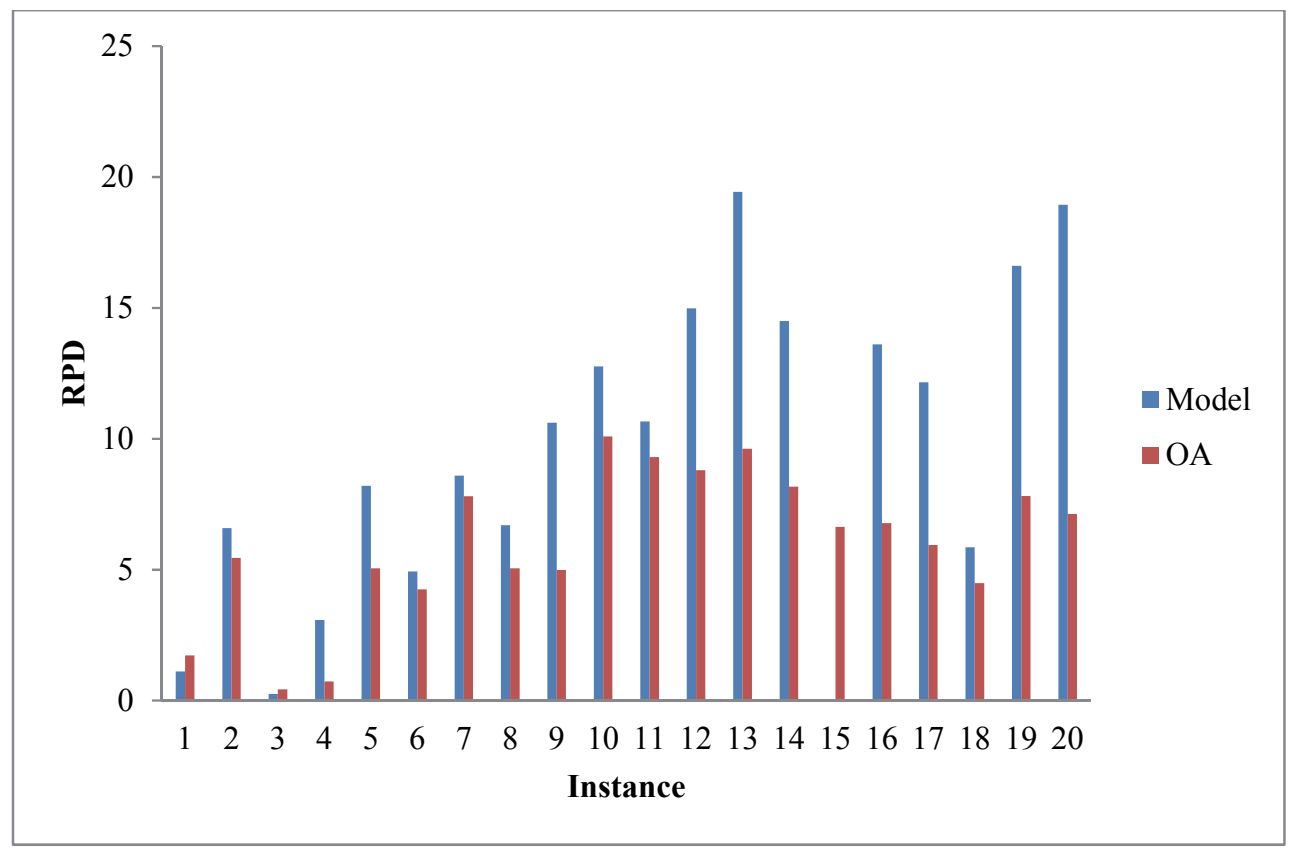

Fig. 2. Comparing the performance of the external approximation algorithms and the mathematical model 
To further investigate the function of the proposed outer approximation algorithm and for managerial analyses in terms of network performance using the proposed outer approximation method, a number of 30 solved examples were obtained and are reported in Table 3. In this table, ND and NV are the number of active distribution centers and vehicles, respectively. Depot utilization and Vehicle utilization are the amount of productivity of distribution centers and vehicles that are defined as a fraction of the total capacity that is used. As seen in this table, generally, the algorithm run time has been increased by increasing the problem size. Also, as is clear from the table, the productivity of distribution centers and vehicles were generally high.

Table 3

Evaluation of the performance of the outer approximation algorithm

\begin{tabular}{|c|c|c|c|c|c|c|c|c|c|c|c|c|c|}
\hline \multirow{2}{*}{ No. } & \multirow{2}{*}{$\mathrm{J}$} & \multirow{2}{*}{$\mathrm{K}$} & \multirow{2}{*}{$\mathrm{V}$} & \multirow{2}{*}{$\begin{array}{l}\text { Solution } \\
\text { time (s) }\end{array}$} & \multirow{2}{*}{ Total cost } & \multirow{2}{*}{ ND } & \multicolumn{3}{|c|}{ Depot utilization } & \multirow{2}{*}{$\mathrm{NV}$} & \multicolumn{3}{|c|}{ Vehicle utilization } \\
\hline & & & & & & & $\max$ & $\min$ & ave & & $\max$ & $\min$ & ave \\
\hline 1 & 10 & 10 & 8 & 252.43 & 42738.74 & 2 & 0.84 & 0.80 & 0.82 & 4 & 0.97 & 0.83 & 0.92 \\
\hline 2 & 10 & 10 & 9 & 285.99 & 42454.50 & 2 & 0.58 & 0.58 & 0.58 & 3 & 0.95 & 0.80 & 0.90 \\
\hline 3 & 10 & 10 & 10 & 368.11 & 42237.03 & 2 & 0.88 & 0.44 & 0.66 & 4 & 0.94 & 0.81 & 0.89 \\
\hline 4 & 10 & 15 & 8 & 449.79 & 73207.06 & 3 & 0.90 & 0.28 & 0.61 & 6 & 0.97 & 0.80 & 0.89 \\
\hline 5 & 10 & 15 & 9 & 504.98 & 86470.38 & 3 & 0.89 & 0.16 & 0.56 & 6 & 0.99 & 0.81 & 0.94 \\
\hline 6 & 10 & 15 & 10 & 517.18 & 60405.47 & 3 & 0.81 & 0.44 & 0.58 & 9 & 0.96 & 0.84 & 0.91 \\
\hline 7 & 10 & 15 & 8 & 482.85 & 57364.87 & 3 & 0.82 & 0.27 & 0.53 & 6 & 0.92 & 0.78 & 0.87 \\
\hline 8 & 10 & 15 & 9 & 536.80 & 61110.79 & 3 & 0.79 & 0.52 & 0.66 & 5 & 0.95 & 0.83 & 0.88 \\
\hline 9 & 10 & 20 & 10 & 565.02 & 78272.50 & 2 & 0.94 & 0.70 & 0.82 & 7 & 0.94 & 0.82 & 0.92 \\
\hline 10 & 10 & 20 & 8 & 576.57 & 91554.16 & 3 & 0.91 & 0.69 & 0.77 & 6 & 0.94 & 0.80 & 0.90 \\
\hline 11 & 10 & 20 & 9 & 630.84 & 81175.34 & 3 & 0.95 & 0.87 & 0.93 & 6 & 0.94 & 0.84 & 0.90 \\
\hline 12 & 10 & 20 & 10 & 543.89 & 73082.08 & 3 & 0.82 & 0.68 & 0.77 & 6 & 0.96 & 0.85 & 0.90 \\
\hline 13 & 10 & 20 & 8 & 725.15 & 67020.52 & 4 & 0.97 & 0.44 & 0.76 & 6 & 0.86 & 0.83 & 0.87 \\
\hline 14 & 15 & 15 & 9 & 847.22 & 52191.71 & 4 & 0.96 & 0.21 & 0.58 & 4 & 0.87 & 0.79 & 0.85 \\
\hline 15 & 15 & 15 & 10 & 682.30 & 50523.26 & 3 & 0.97 & 0.44 & 0.75 & 6 & 0.94 & 0.80 & 0.91 \\
\hline 16 & 15 & 15 & 8 & 749.84 & 51219.77 & 7 & 0.99 & 0.43 & 0.69 & 5 & 0.93 & 0.85 & 0.89 \\
\hline 17 & 15 & 15 & 9 & 513.78 & 43870.24 & 5 & 0.68 & 0.40 & 0.56 & 5 & 0.89 & 0.83 & 0.87 \\
\hline 18 & 15 & 15 & 10 & 531.09 & 49059.02 & 4 & 0.94 & 0.43 & 0.70 & 6 & 0.95 & 0.79 & 0.86 \\
\hline 19 & 15 & 20 & 8 & 597.58 & 73091.39 & 4 & 0.86 & 0.19 & 0.55 & 6 & 0.91 & 0.72 & 0.85 \\
\hline 20 & 15 & 20 & 9 & 705.01 & 80577.41 & 4 & 0.85 & 0.58 & 0.75 & 5 & 0.83 & 0.81 & 0.85 \\
\hline 21 & 15 & 20 & 10 & 855.94 & 68424.06 & 4 & 0.90 & 0.40 & 0.66 & 4 & 0.91 & 0.87 & 0.90 \\
\hline 22 & 15 & 20 & 8 & 791.14 & 76277.11 & 5 & 0.74 & 0.53 & 0.63 & 3 & 0.96 & 0.79 & 0.88 \\
\hline 23 & 15 & 20 & 9 & 803.07 & 68401.22 & 5 & 0.74 & 0.41 & 0.60 & 7 & 0.96 & 0.83 & 0.90 \\
\hline 24 & 20 & 20 & 10 & 1053.17 & 71226.44 & 4 & 0.85 & 0.40 & 0.69 & 5 & 0.88 & 0.77 & 0.85 \\
\hline 25 & 20 & 20 & 8 & 893.80 & 72816.98 & 5 & 0.88 & 0.26 & 0.59 & 6 & 0.95 & 0.85 & 0.90 \\
\hline 26 & 20 & 20 & 9 & 1014.31 & 66940.20 & 5 & 0.90 & 0.15 & 0.52 & 6 & 0.87 & 0.83 & 0.87 \\
\hline 27 & 20 & 20 & 10 & 967.01 & 65959.30 & 5 & 0.82 & 0.12 & 0.52 & 7 & 0.97 & 0.78 & 0.87 \\
\hline 28 & 20 & 20 & 8 & 982.15 & 71191.35 & 5 & 0.77 & 0.42 & 0.63 & 8 & 0.97 & 0.82 & 0.92 \\
\hline 29 & 20 & 20 & 9 & 1088.55 & 60755.51 & 5 & 0.89 & 0.28 & 0.60 & 7 & 0.97 & 0.84 & 0.90 \\
\hline 30 & 20 & 20 & 10 & 776.20 & 67348.93 & 5 & 0.66 & 0.15 & 0.40 & 7 & 0.87 & 0.77 & 0.87 \\
\hline
\end{tabular}

The results of the productivity of the distribution centers and vehicles are given as histogram in Fig. (3) and Fig. (4) in order to better understand the performance of the outer approximation algorithm. 


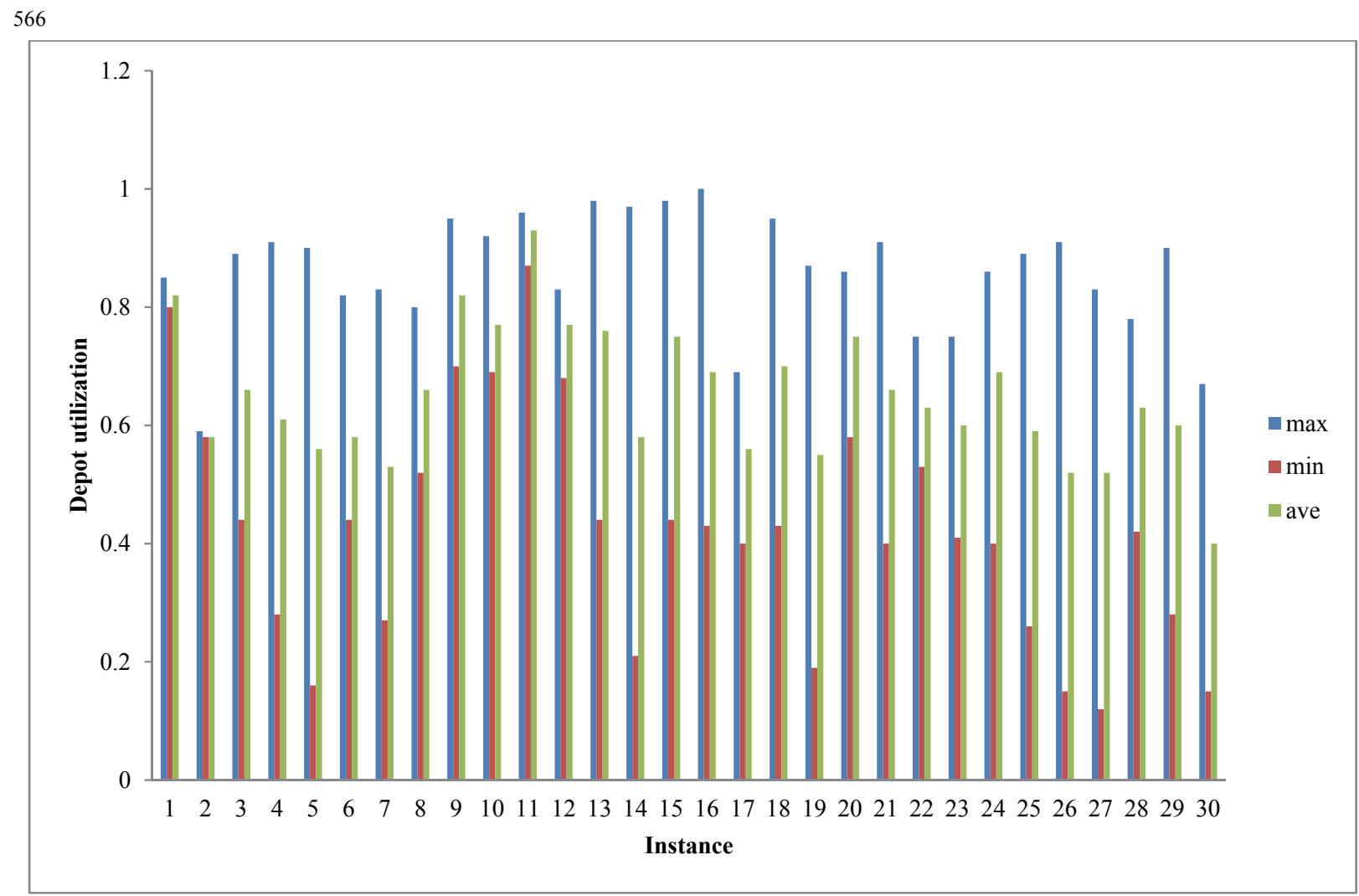

Fig. 3. The using rate of distribution centers

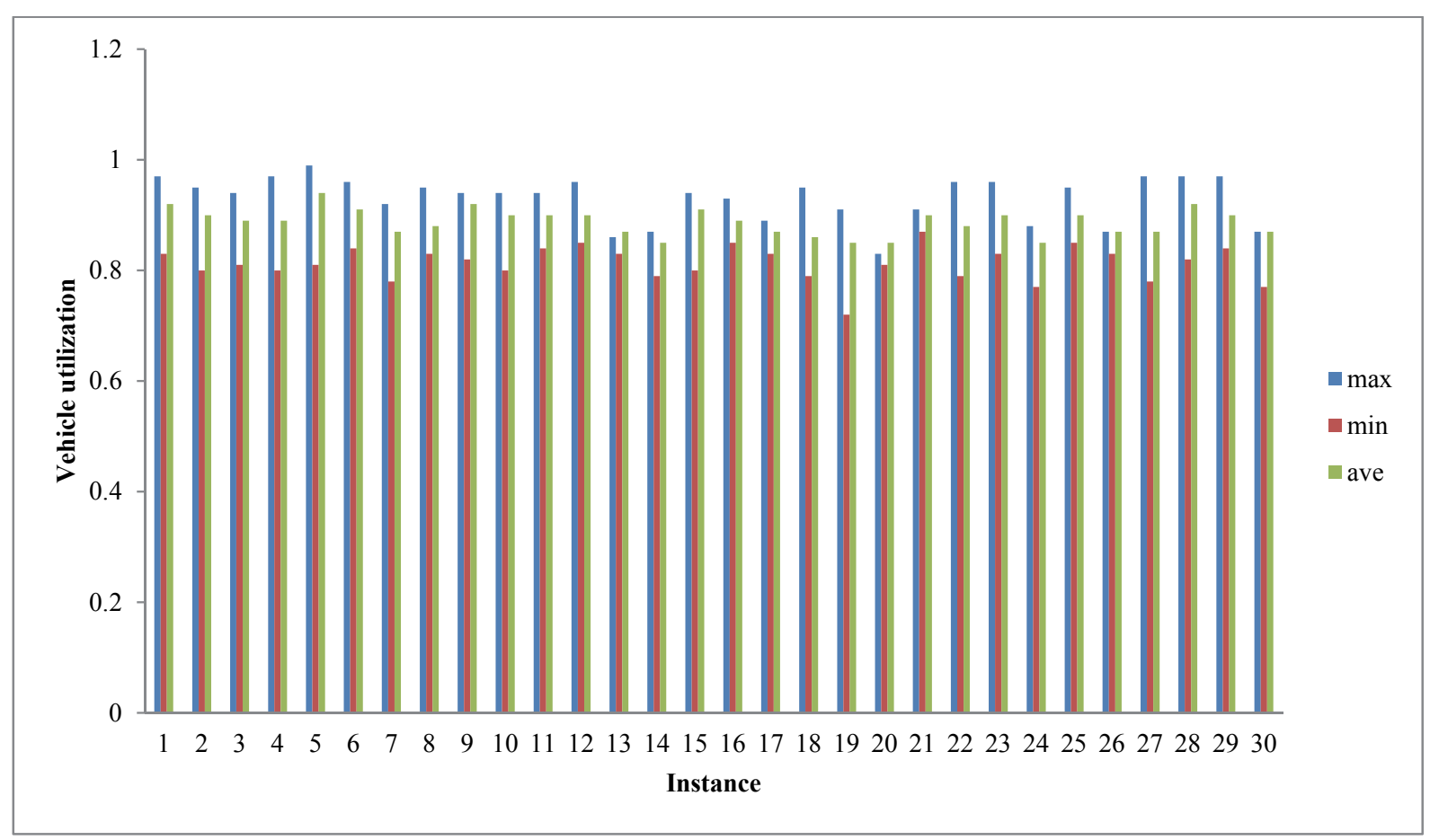

Fig. 4. The using rate of vehicles' capacity 


\section{Conclusions and Recommendations}

In this paper, a new model was developed for the problem of location routing inventory. The proposed model improved the existing models in two main aspects: first, the partial backorder was considered in the present model. Generally, in routing inventory models it is assumed that in the event of a shortage, either all orders are compensated as backorder or total order will be lost. But in real situations, it is probable that only a percentage of orders be lost and the remaining be compensated as the backorder. Thus, in the proposed model by this study, the partial backorder was considered that a part of orders may be lost in it and other part can be compensated as a backorder.

Another major improvement in the proposed model is related to considering disruption risks. It is assumed in the proposed model that distributers be faced with disruption with a low likelihood and this disruption decreases their capacity. So this can cause that the demand of some clients are not completely fulfilled. A nonlinear integer programming model is developed to optimally solve the optimization model. Also, a solution algorithm based on the outer approximation method has been developed for the efficient solution of the problem. The computational results show the effectiveness of the proposed outer approximation method. Specifically, the proposed outer approximation algorithm was generally better than the mathematical model and in the few cases where the mathematical model has had better performance and the performance difference has been negligible. Also, the run time of the outer approximation algorithm was much less than the resolution time of the model. Therefore, the outer approximation algorithm was more efficient than the mathematical model's direct solution.

The research extended the subject's literature from different aspects. However, this study has its limitations that must be addressed in future studies. One of the limitations of the present study is that the problem is considered stationary, but the fact is that customers demand changes during periods, thus, a multi-period dynamic network can be considered for more realization of the studied model. Also taking into account the specific conditions for the possibility of locating facilitating is another approach to develop the present model. In other words, in the real world if the demand of a facility is less than a certain extent, the facility will not be installed because this is not economic. By adding this condition to the proposed model by this research, it is possible to obtain a more realistic model.

\section{Acknowledgement}

The authors would like to thank the anonymous referees for constructive comments on earlier version of this paper.

\section{References}

Adelman, D. (2004). A price-directed approach to stochastic inventory/routing. Operations Research, 52(4), 499-514.

Baita, F., Ukovich, W., Pesenti, R., \& Favaretto, D. (1998). Dynamic routing-and-inventory problems: a review. Transportation Research Part A: Policy and Practice, 32(8), 585-598.

Duran, M. A., \& Grossmann, I. E. (1986). An outer-approximation algorithm for a class of mixedinteger nonlinear programs. Mathematical programming,36(3), 307-339.

Daskin, M. S., Coullard, C. R., \& Shen, Z. J. M. (2002). An inventory-location model: Formulation, solution algorithm and computational results.Annals of operations research, 110(1-4), 83-106.

Fletcher, R., \& Leyffer, S. (1994). Solving mixed integer nonlinear programs by outer approximation. Mathematical programming, 66(1-3), 327-349.

Gaur, V., \& Fisher, M. L. (2004). A periodic inventory routing problem at a supermarket chain. Operations Research, 52(6), 813-822.

Huang, C., Yu, G., Wang, S., \& Wang, X. (2006). Disruption management for supply chain coordination with exponential demand function. Acta Mathematica Scientia, 26(4), 655-669. 
Jaillet, P., Bard, J. F., Huang, L., \& Dror, M. (2002). Delivery cost approximations for inventory routing problems in a rolling horizon framework.Transportation Science, 36(3), 292-300.

Javid, A. A., \& Azad, N. (2010). Incorporating location, routing and inventory decisions in supply chain network design. Transportation Research Part E: Logistics and Transportation Review, 46(5), 582-597.

Kleywegt, A. J., Nori, V. S., \& Savelsbergh, M. W. (2002). The stochastic inventory routing problem with direct deliveries. Transportation Science,36(1), 94-118.

Liu, S. C., \& Lee, S. B. (2003). A two-phase heuristic method for the multi-depot location routing problem taking inventory control decisions into consideration. The International Journal of Advanced Manufacturing Technology, 22(11-12), 941-950.

Liu, S. C., \& Lin, C. C. (2005). A heuristic method for the combined location routing and inventory problem. The International Journal of Advanced Manufacturing Technology, 26(4), 372-381.

Min, H., Jayaraman, V., \& Srivastava, R. (1998). Combined location-routing problems: A synthesis and future research directions. European Journal of Operational Research, 108(1), 1-15.

Nagy, G., \& Salhi, S. (2007). Location-routing: Issues, models and methods.European Journal of Operational Research, 177(2), 649-672.

Shen, Z. J. M., \& Qi, L. (2007). Incorporating inventory and routing costs in strategic location models. European journal of operational research, 179(2), 372-389.

Tavakkoli-Moghaddam, R., Forouzanfar, F., \& Ebrahimnejad, S. (2013). Incorporating location, routing, and inventory decisions in a bi-objective supply chain design problem with riskpooling. Journal of Industrial Engineering International, 9(1), 1-6.

Xuefeng, W. (2010, January). An integrated multi-depot location-inventory-routing problem for logistics distribution system planning of a chain enterprise. In Logistics Systems and Intelligent Management, 2010 International Conference on (Vol. 3, pp. 1427-1431). IEEE.

Yu, Y., Chen, H., \& Chu, F. (2008). A new model and hybrid approach for large scale inventory routing problems. European Journal of Operational Research, 189(3), 1022-1040.

Zhang, B., Ma, Z., \& Jiang, S. (2008, October). Location-routing-inventory problem with stochastic demand in logistics distribution systems. InWireless Communications, Networking and Mobile Computing, 2008. WiCOM'08. 4th International Conference on (pp. 1-4). IEEE.

Zhao, Q. H., Chen, S., \& Zang, C. X. (2008). Model and algorithm for inventory/routing decision in a three-echelon logistics system. European Journal of Operational Research, 191(3), 623-635. 